\title{
Cirugía paliativa mediante operación de Norwood en distintas formas de corazón univentricular: resultados quirúrgicos inmediatos
}

\author{
Pedro Becker R, Patricia Frangini S, G onzalo U rcelay M, \\ Claudio Arretz V, Felipe Heusser R, Pilar Arnaiz G, \\ Francisco G aray G, Manuel J Irarrázaval L, Sergio Morán V, \\ Ricardo Zalaquett S, Gustavo Maturana B, Elisa Castillo $\mathbf{N}^{1}$. \\ Immediate results of palliative \\ surgery for different forms of \\ univentricular heart using the \\ Norwood procedure
}

Background: Norwood procedure is used as the first stage in the palliative treatment of the hypoplastic heart syndrome and can be used, with some technical modifications, in other forms of univentricular heart with aortic stenosis or hypoplasia. These patients have a high mortality (50\%), derived from the procedure itself and from their abnormal physiological status. Aim: To report our experience with the Norwood procedure. Patients and methods: Retrospective analysis of all patients subjected to the Norwood procedure between February 2000 and June 2003. Results: Thirteen patients (9 females, age range 5-60 days and median weight of $3.3 \mathrm{~kg}$ ) were operated. Eight had hypoplastic heart syndrome and five had a single ventricle with aortic arch hypoplasia. The diagnosis was done in utero in eight patients. All technical variations, according to the disposition and anatomy of the great vessels, are described. Cardiac arrest with profound hypothermia was used in all and regional cerebral perfusion was used in nine. Three patients died in the perioperative period and three died in the follow up (two, four and 10 months after the procedure). Gleen and Fontan procedures were completed in five and one patients, respectively. Conclusions: Our results with the Norwood procedure are similar to other series. There is an important mortality in the immediate operative period and prior to the Glenn procedure (Rev Méd Chile 2004; 132: 556-63).

(Key Words: Cardiovascular Surgical Procedures; Heart defects, congenital)

Recibido el 5 de diciembre, 2003. Aceptado el 5 de abril, 2004.

Departamento de Enfermedades Cardiovasculares y Departamento de Pediatría, Facultad de Medicina, Pontificia Universidad Católica de Chile y ${ }^{1}$ Hospital Dr. Sótero del Río.

Correspondencia a: Dr. Pedro Becker R. Marcoleta 367, 6o piso.

Teléfono: 6333030. Fax: 6960108. E-mail: pbecker@med.puc.cl 
$\mathrm{L}$ a operación de Norwood corresponde a un complejo procedimiento, diseñado como etapa inicial en la paliación quirúrgica del síndrome de hipoplasia de corazón izquierdo (SHCI). También es aplicable a otras formas de corazón univentricular asociadas a estenosis, hipoplasia o atresia aórtica. Sus componentes primordiales son: 1) creación de una amplia comunicación entre la arteria pulmonar y la aorta, con la consecuente irrestricta conexión entre el ventrículo único y la "neoaorta"; 2) corregir la coartación aórtica yuxtaductal e hipoplasia del arco; 3) establecer una fuente controlada de flujo sanguíneo pulmonar a través de un shunt sistémico-pulmonar; 4) asegurar la presencia de una amplia comunicación interauricular, de modo de permitir que el retorno venoso pulmonar también sea libre de obstrucción hacia el ventrículo.

El Dr. William Norwood comunicó, en 1980, el primer niño portador de SHCI con sobrevida alejada, luego de una operación inicial paliativa ${ }^{1}$. $\mathrm{Si}$ bien hubo otros intentos para solucionar esta compleja patología, fue Norwood y su grupo, quienes perseveraron perfeccionando el diseño operatorio con modificaciones en $1983^{2}$ y en $1988^{3}$, siendo esta última operación la que hoy se conoce como "Norwood clásico" (Figura 1).

Esta intervención posee una alta mortalidad, la que se explica por lo complejo de la cirugía, por la necesidad de realizarse en el período neonatal, y porque los pacientes permanecen con una fisiología anormal, que es prácticamente idéntica a la que tienen antes de operarse. De hecho, el principal problema es lograr un adecuado balance entre la circulación pulmonar y sistémica, además de preservar una adecuada función ventricular, muchas veces deteriorada transitoriamente después de la cirugía. Por lo tanto, constituye un verdadero desafío de manejo interdisciplinario entre cirujanos, cardiólogos, anestesiólogos e intensivistas.

En nuestro grupo, introdujimos esta técnica el año 2000 y la hemos utilizado para el tratamiento de pacientes con SHCI o con ventrículo único asociado a obstrucción aórtica. Corresponde a la primera etapa en la paliación quirúrgica y va seguida de una segunda etapa, alrededor de los 6 meses de edad (operación de Glenn bidireccional) y por último la tercera etapa entre los 2 y 3 años (operación de Fontan).

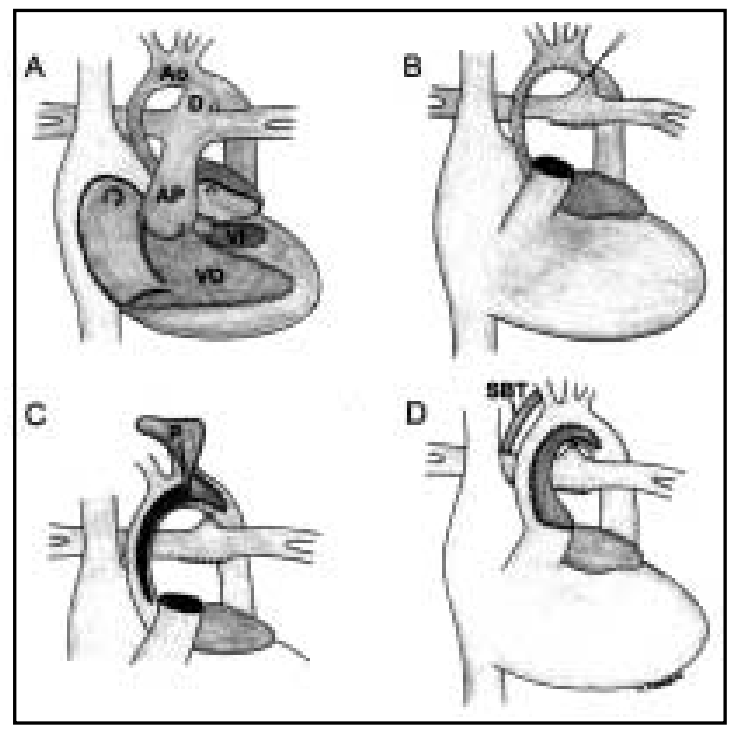

Figura 1. Operación de Norwood clásico. A: hipoplasia de corazón izquierdo con atresia mitral y aórtica, donde el flujo en la aorta depende del ductus. B. Sección de arteria pulmonar. C: Ampliación del arco aórtico con parche de homoinjerto pulmonar. D: Anastomosis entre arteria pulmonar proximal y neoaorta y construcción de shunt de Blalock Taussig. (Ao: aorta. AP: arteria pulmonar. D: ductus. P: parche de homoinjerto. SBT: shunt de Blalock Taussig. VD: ventrículo derecho. VI: ventrículo izquierdo).

El objetivo de este trabajo es comunicar nuestros resultados a tres años de haber incorporado esta técnica quirúrgica al protocolo de tratamiento de pacientes con corazón univentricular.

\section{MATERIAL Y MÉTODO}

Pacientes. Se revisó el registro de los pacientes incorporados al protocolo de corazón univentricular y la base de datos del Servicio de Cirugía Cardíaca, del Hospital Clínico de la Pontificia Universidad Católica de Chile, identificando a todos los pacientes sometidos a operación de Norwood entre febrero de 2000 y junio de 2003.

Se revisaron retrospectivamente fichas clínicas, protocolos operatorios y ecocardiogramas de cada paciente. Todos los que sobrevivieron a la cirugía de Norwood fueron contactados, obteniendo infor- 
mación tanto de sus padres como de sus cardiólogos tratantes, respecto de su estado actual.

Técnica quirúrgica y manejo médico. Los pacientes fueron llevados al pabellón en condiciones hemodinámicas y de equilibrio ácido-base estables, con prostaglandinas y ventilados mayoritariamente con aire ambiental. Todos fueron operados a través de una esternotomía media. La canulación para circulación extracorpórea (CEC) se hizo utilizando una cánula arterial directamente en el ductus o a través de un tubo de politetrafluoretileno (PTFE) conectado a la arteria innominada. Para el retorno venoso se usó una cánula única a través de la orejuela derecha. Una vez en CEC, se indujo hipotermia profunda de $18^{\circ} \mathrm{C}$, tomando las medidas rutinarias para paro circulatorio con hipotermia profunda (PCHP) y perfusión cerebral regional (PCR) (Figura 2). La protección miocárdica se hizo con cardioplejia cristaloide en una dosis única de $30 \mathrm{ml} / \mathrm{kg}$. Se utilizó ultrafiltración convencional durante la CEC, aprotinina y sangre fresca para el cebado de la máquina de corazón-pulmón. El ductus fue ligado al momento de iniciar la CEC y luego seccionado. Se resecó el septum primum a través de la aurícula derecha, de manera de obtener una comunicación interauricular amplia. En la reconstrucción de la neoaorta y arco aórtico, se empleó un parche tomado de homoinjerto pulmonar criopreservado. Se resecó el tejido ductal remanente al momento de reparar la coartación aórtica. Según las variantes anatómicas, se modificó la geometría, disposición y el número de parches necesarios para reconstruir la neoaorta y el arco. Se completó la operación construyendo un shunt sistémico pulmonar tipo
Blalock-Taussig modificado con tubo de PTFE. El esternón se dejó abierto rutinariamente.

Para la salida de CEC, se utilizó un goteo de nitroglicerina y dopamina, agregando selectivamente milrinona o epinefrina. Se intentó obtener una saturación arterial de oxígeno de entre 65 y $75 \%$, con presión parcial de oxígeno sobre 28 mmHg y equilibrio ácido-básico normal.

Estadística. Análisis descriptivo de los resultados expresándolos como medianas con sus respectivos rangos.

\section{Resultados}

Características de los pacientes. Se incluyeron 13 pacientes consecutivos, 9 fueron mujeres (69\%), con una mediana de 12 días de edad (5-60) y 3,3 kilogramos de peso $(2,6-4,7)$.

Ocho pacientes $(62 \%)$ tenían diagnóstico de SHCI, 4 de ellos con atresia mitro-aórtica. De los 5 pacientes restantes, 3 tenían doble entrada de ventrículo único izquierdo, 1 doble salida de ventrículo único derecho y 1 atresia tricuspídea, en todos los casos con la aorta emergiendo del ventrículo hipoplásico.

Ocho pacientes (62\%) tuvieron diagnóstico antenatal, el cual fue confirmado con ecocardiograma realizado inmediatamente después de nacer. En 3 pacientes se solicitó evaluación por cardiólogo debido al hallazgo de soplo en su evaluación en puerperio. Los 2 pacientes restantes ingresaron al servicio de urgencia en shock cardiogénico, requiriendo maniobras de reanima-

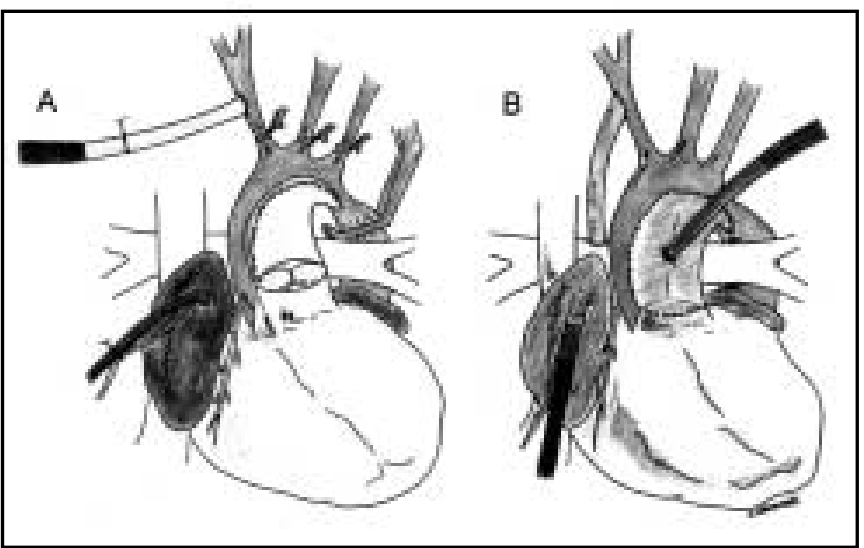

Figura 2. A: Cánula arterial conectada a la arteria innominada a través de un tubo de PTFE, con oclusión proximal de los vasos supraaórticos, perfundiendo a $20-30 \mathrm{ml} / \mathrm{kg} /$ $\min$ a $18^{\circ} \mathrm{C}$. B: Operación de Norwood completada utilizando el mismo tubo de PTFE para construir el shunt de Blalock Taussig. 
ción avanzadas e inicio de infusión de prostaglandinas de urgencia.

La evaluación ecocardiográfica mostró función ventricular derecha cualitativamente conservada en todos los pacientes. La evaluación con Doppler color de la válvula tricúspide reveló insuficiencia leve en 4 pacientes y moderada en 1 . La aorta ascendente midió 5,6 mm $(1,8-7,5)$.

Dos pacientes tenían comunicación interauricular restrictiva, realizándose septostomía de Rashkind.

Los pacientes se mantuvieron monitorizados en la unidad de cuidados intensivos, con infusión de prostaglandinas y sin aporte adicional de oxígeno. Seis pacientes fueron intubados en el preoperatorio para controlar el flujo pulmonar.

Procedimiento quirúrgico. Se realizó cirugía de Norwood clásica en 9 pacientes (69\%) y de Norwood modificado en los otros 4.

Para la construcción del shunt, se empleó un tubo de PTFE de 3,5 $\mathrm{mm}$ en 11 pacientes y de 4 $\mathrm{mm}$ en 2. En la reconstrucción del cayado aórtico se utilizó parche único en 10 pacientes y doble en 3. En las Figuras 3, 4 y 5 se esquematizan las variantes técnicas empleadas.

El tiempo de CEC fue de 202 min (133-340) y el tiempo de oclusión aórtica de 86 min (50-150). En todos los pacientes se usó PCHP, con una duración de 21 min (6-80). En 9 pacientes se usó
PCR, por un tiempo de $63 \mathrm{~min}$ (50-97). En aquellos en quienes se usó esta variante técnica, el tiempo de PCHP fue de $13 \min$ (6-80) en comparación con 62 min (41-104) en quienes no se utilizó.

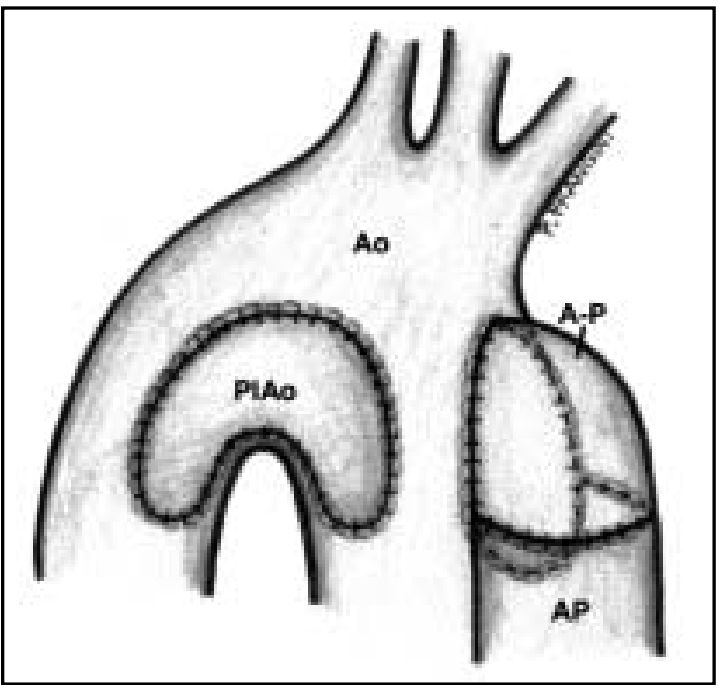

FiguRa 3. Operación de Norwood modificado con arco aórtico derecho y empleo de dos parches independientes. (Ao: aorta. AP: arteria pulmonar. A$\mathrm{P}$ : anastomosis aorto-pulmonar. PlAo: plastia anco aórtico con parche).

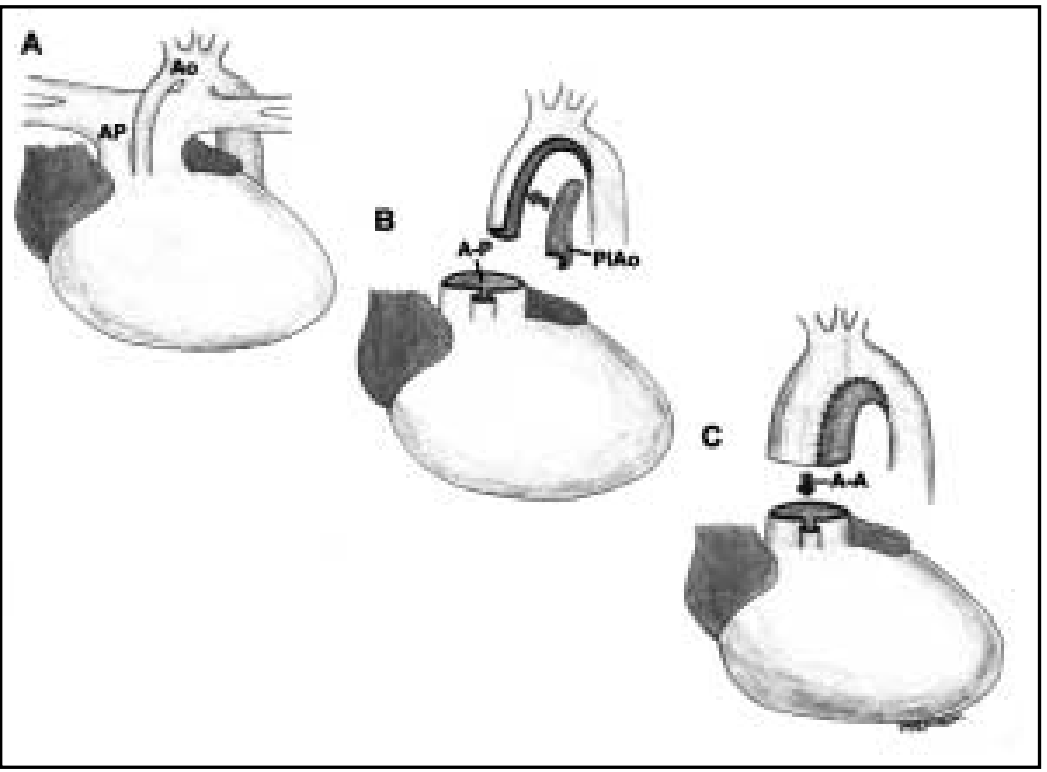

FiguRA 4. Operación de Norwood modificado con mal posición de grandes arterias. (Ao: aorta. AP: arteria pulmonar. A-A: anastomosis aorta-neoaorta término-terminal. A-P: anastomosis aorto-pulmonar látero-lateral. PIAo: plastia arco aórtico con parche). 


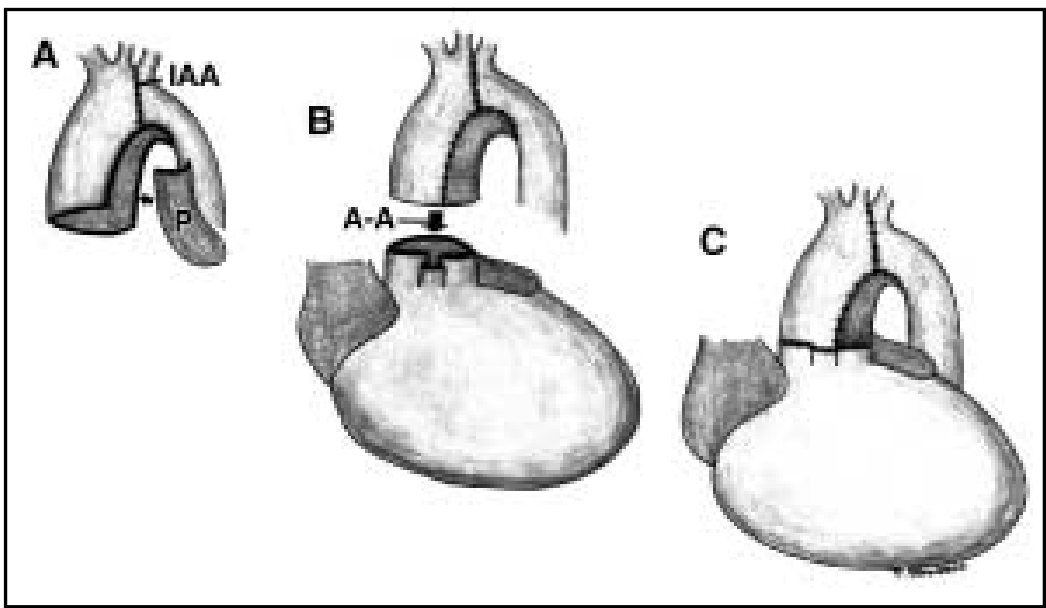

Figura 5. Operación de Norwood modificado con mal posición de grandes arterias e interrupción de arco aórtico tipo B. (A-A: anastomosis aortaneoaorta término-terminal. IAA: interrupción de arco aórtico reparada. P: ampliación de neoaorta con parche).

El cierre esternal diferido se hizo al $4^{\circ}$ día postoperatorio $\left(2^{\circ}-7^{\circ}\right)$.

Resultados perioperatorios. La mortalidad operatoria fue de $23 \%$ (3 pacientes). Un paciente falleció secundario a isquemia miocárdica (aorta ascendente $<2 \mathrm{~mm}$ ); otro falleció súbitamente, aparentemente por embolia aérea coronaria; el último falleció debido a falla multiorgánica secundaria a disfunción miocárdica severa.

Los pacientes recibieron apoyo con drogas vasoactivas intravenosas durante 8 días (7-29). Se usó dopamina en todos, y se asoció en 5 epinefrina y en 5 milrinona.

La duración de la ventilación mecánica fue de 10 días (5-28).

La hospitalización en la unidad de cuidados intensivos fue de 13 días (6-32) y el tiempo de hospitalización total fue de 27 días (17-40).

Las complicaciones quirúrgicas se detallan en la Tabla 1.

\section{Tabla 1. Complicaciones quirúrgicas inmediatas}

\begin{tabular}{|ll}
\hline Reoperación por disfunción del shunt & 1 \\
Reoperación por sangrado & 3 \\
Extubación fallida & 4 \\
Arritmias & 3 \\
Insuficiencia renal aguda & 1 \\
Quilotórax & 2 \\
Enterocolitis necrotizante & 2 \\
Infección intrahospitalaria & 2 \\
\hline
\end{tabular}

$\mathrm{Al}$ momento del alta, la saturación era de $79 \%$ (75-85). Todos fueron dados de alta con aspirina, 9 con digoxina, 9 con furosemida y 6 con captopril.

Seguimiento. Tres pacientes han fallecido (23\%). Uno a los 4 meses, secundario a enterocolitis necrotizante post-cirugía de Glenn bidireccional; otro falleció a los 2 meses en el postoperatorio de una cirugía abdominal electiva; y el restante a los 10 meses, secundario a insuficiencia tricuspídea masiva por endocarditis bacteriana.

Dos pacientes desarrollaron gradiente en el arco aórtico (10 y $60 \mathrm{mmHg}$, respectivamente), requiriendo plastia del arco durante la cirugía de Glenn.

Los 7 pacientes sobrevivientes tienen una mediana de seguimiento de 16 meses (3-36). Tres han completado su etapa de Glenn bidireccional y uno la etapa de Fontan.

\section{DisCUSIÓN}

El SHCI corresponde a una malformación congénita de etiología no precisada, que constituye una de las formas más graves y a la vez más frecuentes de corazón univentriculart ${ }^{4}$ Se caracteriza por la presencia de atresia o estenosis de la válvula mitral, hipoplasia del ventrículo izquierdo, atresia o estenosis de la válvula aórtica, hipoplasia de la aorta ascendente y arco aórtico, y frecuentemente coartación aórtica yuxtaductal. El flujo sistémico 
es mantenido por la presencia de un ductus arterioso persistente amplio.

La evolución natural está marcada por una alta y rápida mortalidad, siendo superior a $80 \%$ al mes de vida ${ }^{5}$. Los esfuerzos para cambiar esta nefasta evolución han sido intensos, constituyendo uno de los mayores desafíos en el ámbito de las cardiopatías congénitas, con resultados que todavía distan de ser óptimos. Por ello, sigue siendo una opción aceptada la actitud "compasiva" con estos pacientes ${ }^{6}$. En nuestro medio, sólo en los últimos cuatro años se ha comenzado a ofrecer tratamiento quirúrgico en forma más o menos sistemática a niños portadores de SHCI, no existiendo reportes en la literatura nacional al respecto.

La operación de Norwood constituye uno de los principales esfuerzos conducentes a paliar el SHCI, pero la mortalidad operatoria es todavía preocupante. Se han descrito varios factores asociados a mal pronóstico, como la inestabilidad hemodinámica preoperatoria, el tamaño diminuto de la aorta ascendente, el compromiso de la función ventricular, la insuficiencia tricuspídea significativa, la prematuridad y el bajo peso de nacimiento ${ }^{5,7-11}$. Al contrario, es un factor de buen pronóstico el tipo de diagnóstico, siendo más favorables, por ejemplo, aquellas patologías que no corresponden a SHCI ${ }^{12-14}$. Por esta razón, en nuestro grupo hemos considerado una contraindicación para realizar esta operación, al menos en esta etapa inicial, la prematuridad, el peso inferior a 2,5 kg, la disfunción ventricular significativa, la insuficiencia tricuspídea significativa, la presencia de drenaje venoso pulmonar anómalo total obstructivo y la presencia de otras malformaciones graves asociadas. De esta manera, hemos contraindicado la cirugía en alrededor de $25 \%$ de los pacientes que nos han referido.

El $62 \%$ de los pacientes tuvo diagnóstico antenatal, lo que constituye un aporte relevante en la estrategia de manejo de estos niños. El diagnóstico antenatal de SHCI puede hacerse desde la vigésima semana de gestación, permitiendo planificar adecuadamente el nacimiento del paciente e implementar oportunamente las medidas tendientes a su estabilización, incluido el inicio precoz de prostaglandinas ${ }^{15-17}$. Cabe mencionar que esta cardiopatía puede pasar inadvertida inicialmente y el paciente debutar con shock cardiogénico a los pocos días de vida, producto del cierre espontáneo del ductus ${ }^{5,15}$. Dos pacientes de nuestra serie requirieron resucitación avanzada por esta causa. Si bien no es fácil hacer una estimación, es muy probable que un número significativo de niños fallezca antes de conocerse el diagnóstico y, por lo tanto, uno de nuestros desafíos es aumentar el diagnóstico antenatal.

En 9 pacientes se realizó lo que mayoritariamente es aceptado hoy como un "Norwood clásico". Creemos que la reconstrucción de la aorta ascendente y arco con homoinjerto, ofrece mejores resultados en cuanto a menor incidencia de recoartación y menor distorsión de la válvula pulmonar en comparación con otras técnicas. Sin embargo, estas diferencias no son tan claras y es lícito inclinarse por la técnica con que el equipo quirúrgico se sienta más cómodo ${ }^{5,15}$. En los otros 4 pacientes se diseñaron variantes hechas a la medida de cada caso individual, pero que conceptualmente corresponden a la misma intervención y por eso la hemos denominado arbitrariamente como "Norwood modificado". Los 2 pacientes que desarrollaron gradiente en el arco aórtico corresponden al primer tercio de la serie; pensamos que esto se produjo debido a redundancia del parche, que generó una invaginación de éste.

El empleo de PCR constituye un interesante aporte técnico de los últimos años, tendiente a disminuir el tiempo de isquemia cerebral ${ }^{18}$. Nosotros reportamos esta técnica en nuestro medio y la hemos implementado en forma rutinaria en la operación de Norwood ${ }^{19}$. Está por verse si efectivamente contribuye a preservar la función cerebral en el mediano y largo plazo.

Las cifras reportadas de mortalidad perioperatoria históricamente han sido del orden de 20 a $30 \%$. Las mejores series han logrado bajar de 10\% pero otras superan el 50\%5,8,10,14,15,20,21. Bartram et $\mathrm{al}^{22}$ realizaron un detallado análisis de las causas de muerte post Norwood, siendo los problemas de irrigación coronaria y aquellos derivados del shunt los más frecuentes. La mortalidad inicial de esta serie es comparable a series internacionales, sin embargo, tiene la importante limitación de ser una serie pequeña.

No es un hecho menor el que estos pacientes permanezcan con una fisiología anormal, con flujo pulmonar dependiente de un shunt sistémico-pulmonar, por lo cual el manejo intra y post operatorio constituye un verdadero desafío ${ }^{23}$. 
Se han incorporado progresivamente diversos métodos para optimizar el manejo postoperatorio, como la adición de dióxido de carbono o nitrógeno a la mezcla de aire, la medición continua de saturación venosa mixta y, recientemente, el uso de fenoxibenzamina con resultados promisonios ${ }^{15,20,24}$. Sin embargo, existe una modificación técnica introducida últimamente, que parece contribuir significativamente en disminuir la mortalidad y facilitar el manejo en la unidad de cuidados intensivos, debido a que el balance entre la circulación pulmonar y sistémica deja de ser crítico ${ }^{5,25}$. Esta modificación consiste en reemplazar el shunt de Blalock-Taussig por uno que conecta el ventrículo y la arteria pulmonar, de modo de obtener sólo flujo sistólico y no sisto-diastólico, como en la primera situación. Si bien existen algunos problemas en relación a esta variante, popularizada por Sano et al, parece ser una de las contribuciones más importantes del último tiempo y por ello nos parece razonable considerar su uso selectivamente.

\section{REFERENCIAS}

1. Norwood W, KirKLin J, Sanders S. Hypoplastic Left heart Syndrome: Experience with palliative surgery. Am J Cardiol 1980; 45: 87-91.

2. Norwood W, Lang P, Hansen D. Physiologic repair of aortic atresia-hypoplastic left heart syndrome. N Engl J Med 1983; 308: 23-6.

3. Pigott J, Murphy J, Barber G, Norwood W. Palliative reconstructive surgery for hypoplastic left heart syndrome. Ann Thorac Surg 1988; 45: 122-8.

4. FYleR D. Report of The New England Regional Infant Cardiac Program. Pediatrics 1980; 65: 375.

5. Kirkun J, BarRat-Boyes B. Aortic Atresia and Other Forms of Hypoplastic Left Heart Physiology. En: Kirklin J, Barrat-Boyes B: Cardiac Surgery, ed 3. New York: Churchill Livingstone, 2003; 1377-400.

6. Osiovich H, Phiшipos E, Byrne P, Robertson M. Hypoplastic Left Heart Syndrome: "To treat or not to treat". Journal of Perinatology 2000; 20: 363-5.

7. Jonas R, Hansen D, Cook N, Wessel D. Anatomic subtype and survival after reconstructive operation for hypoplastic left heart syndrome. J Thorac Cardiovasc Surg 1994; 107: 1121-8.

8. IAnNetToni M, Bove E, Mosca R, Lupinetti $\mathrm{F}$, DOROSTKAR P, LUDOMIRSKY A ET AL. Improving results
El riesgo de muerte se prolonga en el mediano plazo, especialmente antes de realizar la operación de Glenn ${ }^{5,10,15,26}$. En nuestra experiencia un paciente falleció antes del Glenn y dos antes del Fontan. Existe evidencia no publicada de que la modificación de Sano podría también contribuir a disminuir esta atrición previa a la segunda etapa.

Esta serie inicial representa otra contribución al manejo del corazón univentricular en nuestro medio y debiera ayudar a que más pacientes logren finalmente llegar a la operación de Fontan, que es la "vía final común" de todos estos niños ${ }^{27}$, sobre todo si asumimos que el SHCI es, en la actualidad, una de las principales formas de corazón univentricular. La posibilidad de incorporar la operación de Norwood en nuestro medio constituye, a nuestro juicio, un paso importante en el desarrollo de la cardiología y cardiocirugía pediátrica en Chile y en ello radica el principal aporte de esta publicación.

with first stage palliation for hypoplastic left heart syndrome. J Thorac Cardiovasc Surg 1994; 107: 934-40.

9. Forbess J, Cook N, Roth S, Serraf A, Mayer J, Jonas $\mathrm{R}$. Ten years institutional experience with palliative surgery for hypoplastic left heart syndrome. Circulation 1995; 92 (suppl II): II-262-II-266.

10. Mahle W, Spray T, Wernovsky G, Gaynor W, Ciark B. Survival After Reconstructive Surgery for Hypopastic Left Heart Syndrome. A 15 years experience from a single institution. Circulation 2000; 102 (suppl III): III-136-III-141.

11. Ashburn D, McCrindie B, Tchervenkov C, Jacobs M, Lofland G, Bove E et al. Outcomes after Norwood operation in neonates with critical aortic stenosis or aortic atresia. J Thorac Cardiovasc Surg 2003; 125: 1070-82.

12. Kanter K, Miluer B, Cuadrado A, Vincent R. Successful Application of the Norwood Procedure for Infants without Hypoplastic Left Heart Syndrome. Ann Thorac Surg 1995; 59: 301-4.

13. Jacobs M, Rychik J, Murphy J, Nicolson S, Steven J, NoRwood W. Results of Norwood's operation for lesions other than hypoplastic left heart syndrome. J Thorac Cardiovasc Surg 1995; 110: 155562. 
14. Daebritz S, Nolert G, Zurakowski D. Results of Norwood Stage I operation: Comparison of hipoplastic left heart syndrome with other malformations. J Thorac Cardiovasc Surg 2000; 199: 358-67.

15. Dandolu B, Jacobs M. Recent Advances in Reconstructive Surgical Management of Hypoplastic Left Heart Syndrome. En: Advances in Cardiac Surgery, vol 13. St Louis: Mosby, 2001; 21-60.

16. Brackley K, Kilby M, Wright J, Brawn W, Sethin B, STUMPER O ET AL. Outcome after prenatal diagnosis of hypoplastic left heart syndrome. Lancet 2000; 356: 1143-7.

17. Tworetzky W, McElhinney D, Reddy M, Brook M, Hanley F, Silverman N. Improved surgical outcomes after fetal diagnosis of hypoplastic left heart syndrome. Circulation 2001; 103: 1269-73.

18. Pigula F, Siewers R, Nemoto E. Regional perfusion of the brain during neonatal arch reconstruction. J Thorac Cardiovasc Surg 1999; 117: 1023-4.

19. Becker P, Bahamondes J, Arretz C, Fajardo C. Perfusión cerebral regional en recién nacidos sometidos a reconstrucción del arco aórtico: descripción de la técnica en dos entidades diferentes. Rev Chil Cardiol 2002; 21: 84-90.

20. Tweddell J, Hoffman G, Fedderly R, Berger S, Thomas J, Ghanayem N et al. Phenoxybenzamine improves systemic oxygen delivery after the Norwood procedure. Ann Thorac Surg 1999; 67: 161-8.
21. Jacobs M, Biackstone E, Bailey L, The Congenital HEART SURGEONS SOCIETY. Intermediate survival in neonates with aortic atresia: a multi-institutional study. J Thorac Cardiovasc Surg 1998; 116: 417-31.

22. Bartram U, Grünenfelder J, Van Praagh R. Causes of Death After the Modified Norwood Procedure: A Study of 122 Postmortem Cases. Ann Thorac Surg 1997; 64: 1795-802.

23. Barnea O, Austin E, Richman B, Santamore W. Balancing the Circulation: Theoretic optimization of pulmonary/systemic flow ratio in hypoplastic left heart syndrome. JACC 1994; 24: 1376-81.

24. Bradiey S, Simsic J, Atz A. Hemodynamic Effects of Inspired Carbon Dioxide after the Norwood Procedure. Ann Thorac Surg 2001; 72: 2088-94.

25. Sano S, Ishino K, Kawada M, Arai S, Kasahara S, AsA T ET AL. Right ventricle-pulmonary shunt in first stage palliation of hipoplastic left heart syndrome. J Thorac Cardiovasc Surg 2003; 126: 504-10.

26. Wiwhams D, Geujns A, Moskowitz A, Weinberg A, Ng J, Crawford E et al. Hypoplastic Left Heart Syndrome: valuing the survival. J Thorac Cardiovasc Surg 2000; 119: 720-31.

27. Becker P, Frangini P, Urceiay G, CastiLo M, Heusser F, ARNaIz P ET AL. Operación de Fontan: resultados inmediatos y a mediano plazo con anastomosis cavopulmonar total. Rev Méd Chile 2002; 130: 1217-26. 